\section{Near miss neonatal precoce identificado com base em sistemas de informação em saúde}

\author{
Early neonatal near miss identified through \\ health information systems
}

Near miss neonatal precoz identificado según el
sistema de información en salud

\section{Resumo}

O objetivo foi validar uma definição de identificação de casos de near miss neonatal precoce utilizando dados dos sistemas de informação em saúde (SIS). Estudo de validação concorrente entre três definições para identificação de casos de near miss neonatal precoce, realizado em hospital universitário com nascidos vivos ocorridos em 2012. Foram aplicadas três definições a esta coorte de nascidos vivos com utilização dos critérios peso ao nascer, idade gestacional, Índice de Apgar no 50 minuto de vida, internação em Unidade de Terapia Intensiva neonatal, ventilação mecânica e más-formações congênitas com diferentes combinações, considerando as proposições de dois artigos brasileiros publicados (definição Silva et al.; definição Pillegi-Castro et al.) e uma terceira (definição SIS) com dados disponíveis em Sistemas de Informação em Saúde. Foram considerados casos os sobreviventes às condições de risco até o 7 o dia de vida. Para a validação concorrente, adotaram-se como referência os óbitos neonatais precoces. Dos 2.097 nascidos vivos estudados, 33 foram a óbito no período neonatal precoce, e o número de casos de near miss neonatal precoce variou segundo a definição adotada: 153 (definição Silva), 194 (definição Pileggi-Castro) e 304 (definição SIS). A sensibilidade e especificidade foi, respectivamente, 97\% e 92,6\% na definição Silva, 90,9\% e 90,6\% na definição Pileggi-Castro e 93,9\% e 85,3\% na definição SIS. Os resultados mostram que a definição SIS apresenta sensibilidade e especificidade próxima às outras definições e sugere que é possível monitorar o near miss neonatal precoce com uso apenas de dados disponíveis nos sistemas oficiais de informações em saúde.

Near Miss; Recém-Nascido; Mortalidade Neonatal Precoce; Estatísticas Vitais; Sistemas de Informação
Karla Eveline Ximenes de França 1 Mirella Bezerra Rodrigues Vilela 1

Paulo Germano de Frias 2

Gabriela da Silveira Gaspar 1

Silvia Wanick Sarinho 1

doi: 10.1590/0102-311X00167717

\author{
Correspondência \\ K. E. X. França \\ Universidade Federal de Pernambuco. \\ Av. Professor Moraes Rego s/n, Prédio das Pós-graduações do \\ CCS, 10 andar, Cidade Universitária, Recife, PE 50670-420, \\ Brasil. \\ karla_ximenes@hotmail.com \\ 1 Universidade Federal de Pernambuco, Recife, Brasil. \\ 2 Instituto de Medicina Integral Professor Fernando Figueira, \\ Recife, Brasil.
}




\section{Introdução}

O termo near miss refere-se a casos de quase morte decorrentes de alguma complicação severa, mas que sobrevivem 1,2. Ao identificar recém-nascidos que escaparam da morte, as deficiências nos serviços ofertados à saúde do binômio mãe-bebê podem ser detectadas e localizadas, levando a uma melhora na assistência 3. Para o near miss materno, conceitos e critérios operacionais foram desenvolvidos, validados e posteriormente recomendados pela Organização Mundial da Saúde (OMS) 4. Entretanto, para o período neonatal não existe consenso apropriado com a finalidade de identificar os casos 2,5.

Estudos que desenvolveram definições para diferenciar os casos near miss neonatal 1,3,5,6,7 são insuficientes e a maioria utilizou o período neonatal precoce 1,3,5,7. Para garantir sua operacionalidade, maior utilidade e oportunidade, dois obstáculos precisam ser superados: o estabelecimento de consenso conceitual e a busca de alternativas para uso em larga escala no cotidiano dos sistemas de saúde. Com o aprofundamento das discussões sobre o near miss neonatal, publicações se multiplicam, contudo seu uso, em geral, se restringe às pesquisas.

$\mathrm{O}$ aproveitamento em maiores contingentes populacionais pode ser facilitado ao se dispor de dados em sistemas de informação em saúde (SIS). No Brasil, há sistemas oficiais de registro contínuo com uma multiplicidade de dados sobre eventos vitais e morbidade com bom nível de adequação das informações, além de acessíveis 8,9 .

A identificação de casos near miss neonatal pela base de dados de registro contínuo associada a sua vigilância favorece a compreensão sobre os determinantes da morbimortalidade, as circunstâncias das ocorrências e as fragilidades assistenciais dos serviços de saúde, que culminam em desfechos diversos, favoráveis ou desfavoráveis, o que contribuirá para o aperfeiçoamento da assistência à gestante e ao recém-nascido, facilitando o alcance de metas pactuadas internacionalmente 2,10,11.

Estudos comparativos entre diferentes definições para identificação de casos de near miss neonatal podem enriquecer o debate em curso. Este artigo objetivou validar uma definição de identificação de casos de near miss neonatal precoce com base nos sistemas oficiais de informação em saúde.

\section{Métodos}

Estudo de validação concorrente realizado no Hospital das Clínicas (HC) da Universidade Federal de Pernambuco, Município de Recife, Pernambuco, Brasil, que realiza em média 150 partos por mês e é referência para gestação e parto de alto risco 12 .

Foram estudados os nascidos vivos no ano de 2012 que apresentaram qualquer uma das condições de risco ao nascer, descritas em cada definição, e que permaneceram vivos até o 7o dia de vida (Tabela 1). Quanto à definição Silva et al. 6, o período de sobrevivência dos recém-nascidos com ameaça à vida foi adaptado para o período neonatal precoce visto que a proposta da pesquisa foi trabalhar com o componente mais crítico da mortalidade infantil 10. A definição Pileggi-Castro et al. 5 foi adaptada, restringindo-a exclusivamente aos critérios pragmáticos para possibilitar a coleta das informações nos sistemas de informações em saúde. A definição proposta pelo nosso estudo (SIS) acrescentou o critério internação em Unidade de Terapia Intensiva neonatal (UTI neonatal) por indicar que os recém-nascidos possivelmente enfrentaram situações de maior gravidade.

Os dados referentes aos nascidos vivos e os óbitos neonatais precoces foram extraídos, respectivamente, do Sistema de Informações sobre Nascidos Vivos (SINASC) e do Sistema de Informações sobre Mortalidade (SIM). O dado sobre uso de ventilação mecânica foi obtido no Censo da Unidade Neonatal e, para obter a informação para o critério internação em UTI neonatal, foram usados dados do Sistema de Informações Hospitalares do Sistema Único de Saúde (SIH-SUS) por meio da busca do código 08.02.01.012-1, referente à Diária de UTI neonatal TIPO II. A coleta e a análise dos dados ocorreram no período de outubro de 2015 a maio de 2016.

Para verificar a confiabilidade das variáveis do SINASC, peso ao nascer, idade gestacional, Índice de Apgar no 5o minuto de vida, presença de más-formações congênitas (campo fechado da Declaração de Nascido Vivo - DNV), e do SIH-SUS, internação em UTI neonatal, foram selecionados probabilisticamente 60 prontuários da unidade neonatal do HC, do ano de 2012. Para as variáveis numéricas, foi aplicado o coeficiente de correlação intraclasse (CCI) e se utilizou o coeficiente de concordância 
Tabela 1

Definições para a identificação de casos de near miss neonatal precoce.

\begin{tabular}{|c|c|c|c|c|c|c|}
\hline Definições & $\begin{array}{c}\text { Idade } \\
\text { gestacional } \\
\text { (semanas) }\end{array}$ & $\begin{array}{c}\text { Peso ao } \\
\text { nascer }(g)\end{array}$ & $\begin{array}{l}\text { Apgar no 5o } \\
\text { minuto de vida }\end{array}$ & $\begin{array}{l}\text { Ventilação } \\
\text { mecânica }\end{array}$ & $\begin{array}{l}\text { Más-formações } \\
\text { congênitas }\end{array}$ & $\begin{array}{c}\text { Internação em UTI } \\
\text { neonatal }\end{array}$ \\
\hline Silva * & $<32$ & $<1,500$ & $<7$ & Sim & Sim & - \\
\hline Pileggi-Castro ** & $<33$ & $<1,750$ & $<7$ & - & - & - \\
\hline SIS $* \star \star$ & $<33$ & $<1,750$ & $<7$ & - & - & Sim \\
\hline
\end{tabular}

SIS: sistemas de informação em saúde; UTI: Unidade de Terapia Intensiva.

* Definição proposta por Silva et al. 6, adaptada para o período neonatal precoce;

** Definição validada por Pileggi-Castro et al. 5, adaptada exclusivamente para critérios pragmáticos;

*** Definição proposta pelo estudo.

kappa para as variáveis categóricas. Adotou-se como adequado um resultado a partir de 0,61, o que representa uma concordância substancial para o kappa 13, e de satisfatória a boa para o CCI 14 .

As definições Silva, Pileggi-Castro e SIS foram aplicadas numa população de 2.097 nascidos vivos, pelo SINASC, para identificar os recém-nascidos que se enquadravam nos critérios. Para os critérios internação em UTI neonatal e uso de ventilação mecânica, os recém-nascidos foram identificados, respectivamente, pelo SIH-SUS e pelo censo da unidade neonatal do HC, e em seguida foi realizada uma busca manual para localizar o número da DNV e as informações desses recém-nascidos no SINASC (a busca foi feita pela variável nome da mãe e a confirmação pelo sexo da criança e data de nascimento). Como produto dessa etapa, foram construídos três bancos (Silva, $\mathrm{n}=185$; Pileggi-Castro $\mathrm{n}=224$; SIS, $\mathrm{n}=335$ ) com dados do SINASC dos recém-nascidos que apresentaram as condições ameaçadoras de vida estudadas.

Para identificar os recém-nascidos que foram a óbito no período neonatal precoce dentre os que se enquadravam nos critérios das definições Silva, Pileggi-Castro e SIS, realizou-se um linkage determinístico entre os bancos com informações do SINASC gerados na etapa anterior e o SIM, utilizando como campo de busca o número da DNV que consta na Declaração de Óbito (DO), e a confirmação aconteceu pela variável nome da mãe. Ao final, esses recém-nascidos que morreram (Silva, $\mathrm{n}=32$; Pileggi-Castro, $\mathrm{n}=30$; SIS, $\mathrm{n}=31$ ) foram excluídos dos bancos de dados para assim obter os sobreviventes, classificados como casos de near miss neonatal precoce conforme as definições Silva (153 casos), Pileggi-Castro (194 casos) e SIS (304 casos), que constituíram o universo de casos desta pesquisa (Figura 1).

Para comparar o desempenho das três definições mediante validação concorrente, foram usados como referência os óbitos neonatais precoces conforme os artigos originais 5,6 . Foram calculados sensibilidade, especificidade e valores preditivos positivo e negativo para cada definição, e a comparação dos valores foi feita por intermédio do intervalo de $95 \%$ de confiança (IC95\%).

O processamento e a análise dos dados foram feitos pelos programas Microsoft Excel 2010 (Microsoft Corp., Estados Unidos), BioEstat 5.3 (Sociedade Civil Mamirauá, Manaus, Brasil) e Epi Info versão 6.04d (Centers for Disease Control and Prevention, Atlanta, Estados Unidos).

A pesquisa recebeu aprovação do Comitê de Ética em Pesquisa sob o número do parecer 1.226.298 e CAAE: 47358315.1.0000.5208.

\section{Resultados}

Os resultados do teste de confiabilidade realizado entre os dados dos Sistemas de Informação em Saúde (SINASC e SIH-SUS) e dos prontuários podem ser vistos na Tabela 2. O conjunto das variáveis foi considerado confiável, com destaque para índice de Apgar no 5o minuto de vida, presença de más-formações congênitas e internação em UTI neonatal que obtiveram correlação/ concordância perfeita. 
Figura 1

Fluxograma de processamento dos dados. Hospital das Clínicas, Recife, Pernambuco, Brasil, 2012.

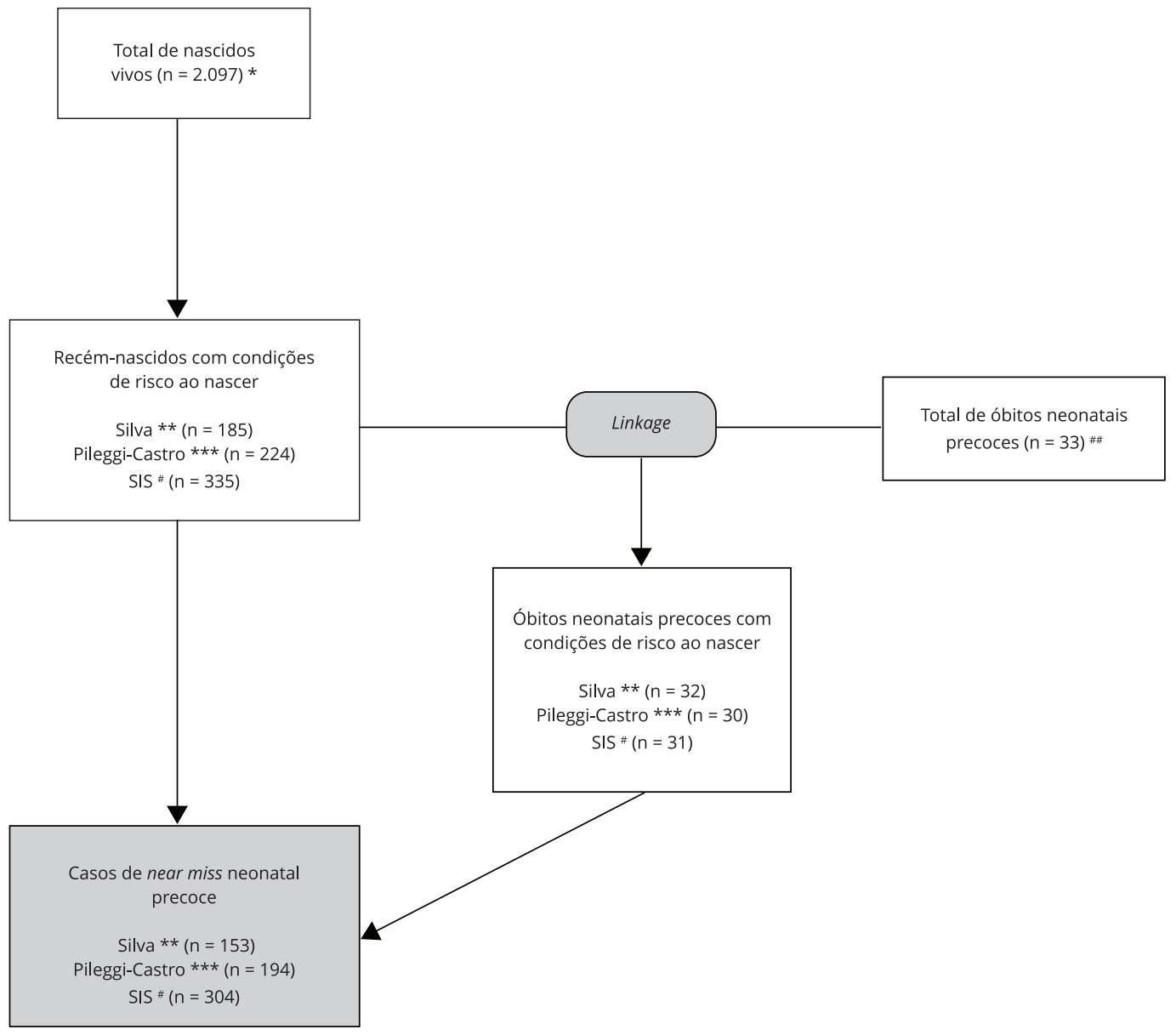

SIS: sistemas de informação em saúde.

* Fonte: Sistema de Informações sobre Nascidos Vivos;

** Definição proposta por Silva et al. 6, adaptada para o período neonatal precoce;

*** Definição validada por Pileggi-Castro et al. 5, adaptada exclusivamente para critérios pragmáticos;

\# Definição proposta pelo estudo;

\#\# Sistema de Informações sobre Mortalidade.

Do total de 2.097 nascidos vivos no HC, no ano de 2012, 33 foram a óbito no período neonatal precoce e 153 foram classificados como casos de near miss neonatal precoce de acordo com a definição Silva, 194 de acordo com a definição Pileggi-Castro e 304 de acordo com a definição SIS (Figura 1).

Nas definições Silva e Pileggi-Castro, o critério idade gestacional foi o que isoladamente mais concentrou casos de near miss neonatal precoce, diferentemente do observado na definição SIS, em que o critério de entrada que mais se destacou foi internação em UTI neonatal (Tabela 3).

A validação concorrente demonstrou que não houve diferença no valor da sensibilidade das três definições quando comparados pelo IC95\%. A especificidade variou de 85,3\% (IC95\%: 83,7-86,8) na definição SIS a 92,6\% (IC95\%: 91,4-93,7) na definição Silva (Tabela 4). 
Tabela 2

Confiabilidade das variáveis nos sistemas de informação e nos prontuários. Hospital das Clínicas, Recife, Pernambuco, Brasil, 2012.

\begin{tabular}{lcc}
\hline Critérios & Teste & Valor de $\mathbf{p ~}$ \\
\hline Variáveis numéricas & Coeficiente de correlação intraclasse & $<0,0001$ \\
Peso ao nascer * & 0,834 & $<0,0001$ \\
Idade gestacional * & 0,662 & $<0,0001$ \\
Índice de Apgar no 5o minuto de vida * & 1,0 & $<0,0001$ \\
Variáveis categóricas & Coeficiente de concordância Kappa & $<0,0001$ \\
Presença de más-formações congênitas * & 1,0 & 1,0 \\
Internação em UTI neonatal ** & & \\
\hline
\end{tabular}

UTI: Unidade de Terapia Intensiva.

* Sistema de Informações sobre Nascidos Vivos (SINASC);

** Sistema de Informações Hospitalares (SIH).

\section{Tabela 3}

Caracterização dos casos de near miss neonatal precoce por critério de entrada (exclusivamente por cada critério *). Hospital das Clínicas, Recife, Pernambuco, Brasil, 2012.

\begin{tabular}{|c|c|c|}
\hline Definição & $\mathbf{n}$ & $\% * \star$ \\
\hline \multicolumn{3}{|l|}{ Silva $(n=153)$} \\
\hline Idade gestacional < 32 semanas & 40 & 26,14 \\
\hline Peso ao nascer $<1.500 \mathrm{~g}$ & 27 & 17,65 \\
\hline Apgar no $5^{\circ}$ minuto $<7$ & 21 & 13,72 \\
\hline Presença de más-formações congênitas & 4 & 2,61 \\
\hline Utilização de ventilação mecânica & 3 & 1,96 \\
\hline \multicolumn{3}{|l|}{ Pileggi-Castro $(n=194)$} \\
\hline Idade gestacional < 33 semanas & 51 & 26,29 \\
\hline Peso ao nascer $<1.750 \mathrm{~g}$ & 48 & 24,74 \\
\hline Apgar no 5 o minuto $<7$ & 26 & 13,40 \\
\hline \multicolumn{3}{|l|}{ SIS (n = 304) } \\
\hline Internação em UTI neonatal & 110 & 36,18 \\
\hline Idade gestacional $<33$ semanas & 37 & 12,17 \\
\hline Peso ao nascer $<1.750 \mathrm{~g}$ & 28 & 9,21 \\
\hline Apgar no 5 o minuto $<7$ & 12 & 3,95 \\
\hline
\end{tabular}

SIS: sistemas de informação em saúde; UTI: Unidade de Terapia Intensiva.

* Casos classificados como near miss neonatal precoce apenas por 1 critério;

** Percentual total de casos de near miss neonatal precoce segundo cada definição: Silva et al. 6; Pileggi-Castro et al. 5;

SIS, proposta pelo estudo.

\section{Discussão}

Os casos de near miss neonatal precoce podem ser identificados por meio dos SIS de modo simplificado e com elevada sensibilidade e especificidade devido à melhora na cobertura e qualidade dos dados do Brasil 8,15. Como a pesquisa utilizou dados secundários, há possibilidade de problemas relacionados à qualidade da fonte. No entanto, a completude das informações dos critérios utilizados se 
Tabela 4

Validação concorrente: desempenho das definições de near miss neonatal precoce com intervalos de 95\% de confiança (IC95\%). Hospital das Clínicas, Recife, Pernambuco, Brasil, 2012.

\begin{tabular}{|c|c|c|c|c|c|c|}
\hline \multirow[t]{4}{*}{$\begin{array}{l}\text { Near miss neonatal } \\
\text { precoce }\end{array}$} & \multicolumn{2}{|c|}{$\begin{array}{c}\text { Óbitos neonatais } \\
\text { precoces }\end{array}$} & \multirow[t]{4}{*}{$\begin{array}{c}\text { Sensibilidade } \\
\qquad a /(a+c)\end{array}$} & \multirow[t]{4}{*}{$\begin{array}{l}\text { Especificidade } \\
\qquad d /(b+d)\end{array}$} & \multirow[t]{4}{*}{$\begin{array}{l}\text { Valor preditivo positivo } \\
\qquad a /(a+b)\end{array}$} & \multirow[t]{4}{*}{$\begin{array}{l}\text { Valor preditivo negativo } \\
\qquad d /(c+d)\end{array}$} \\
\hline & + & - & & & & \\
\hline & $+a$ & b & & & & \\
\hline & $-c$ & d & & & & \\
\hline \multirow[t]{3}{*}{ Silva * } & 32 & 153 & $97,0 \%$ & $92,6 \%$ & $17,3 \%$ & $99,9 \%$ \\
\hline & & & $(82,5-99,8)$ & $(91,4-93,7)$ & $(12,3-23,7)$ & $(99,7-100,0)$ \\
\hline & 1 & 1.911 & & & & \\
\hline \multirow[t]{3}{*}{ Pileggi-Castro ** } & 30 & 194 & $90,9 \%$ & $90,6 \%$ & $13,4 \%$ & $99,8 \%$ \\
\hline & & & $(74,5-97,6)$ & $(89,2-91,8)$ & $(9,4-18,7)$ & $(99,5-100,0)$ \\
\hline & 3 & 1.870 & & & & \\
\hline \multirow[t]{3}{*}{ SIS *** } & 31 & 304 & $93,9 \%$ & $85,3 \%$ & $9,3 \%$ & $99,9 \%$ \\
\hline & & & $(78,4-98,9)$ & $(83,7-86,8)$ & $(6,5-13,0)$ & $(99,5-100,0)$ \\
\hline & 2 & 1.760 & & & & \\
\hline
\end{tabular}

* Definição proposta por Silva et al. 6, adaptada para o período neonatal precoce;

** Definição validada por Pileggi-Castro et al. 5, adaptada exclusivamente para critérios pragmáticos;

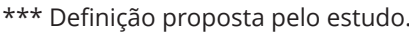

mostrou excelente (100\%), e para avaliar a confiabilidade, confrontaram-se os dados disponíveis nos SIS e nos prontuários, que demostraram resultados favoráveis. O menor valor encontrado foi o CCI para a variável idade gestacional, possivelmente prejudicado pelo método de medida 16: a data da última menstruação é o método prioritário para a estimativa da idade gestacional no SINASC, enquanto na unidade neonatal do HC, o método utilizado é o Capurro.

Uma limitação dos estudos na temática do near miss neonatal é a dificuldade da aplicação do conceito em hospitais de diferentes complexidades. A gravidade da doença ou condição de risco é possivelmente um importante fator de confusão quanto à associação do near miss neonatal com serviços de saúde de complexidades tecnológicas distintas 6,7. Ademais, a fonte da informação internação em UTI neonatal da definição SIS é o SIH do Sistema Único de Saúde. Diante disso, recomenda-se o uso da definição proposta por este estudo, a priori, apenas em hospitais terciários com UTI neonatal, que prestam serviço ao SUS.

A definição SIS foi a que identificou maior número de casos como near miss neonatal precoce (304), com o critério internação em UTI neonatal sendo aquele que isoladamente mais contribuiu na identificação, diferentemente das demais definições em que predominou a idade gestacional seguida pelo peso ao nascer. Estes últimos critérios são fortemente relacionadas às principais causas de óbito neonatal precoce 10,17 e refletem possivelmente fragilidades na assistência pré-natal e na atenção ao parto 18,19 .

Os casos identificados exclusivamente pelo critério internação em UTI neonatal na definição SIS apresentavam peso ao nascer $\geq 1,750 \mathrm{~g}$, idade gestacional $\geq 33$ semanas e índice de Apgar no $5 \underline{\text { o }}$ minuto $\geq 7$, sugerindo que a necessidade decorreu também de outros motivos, o que agrega valor ao apontar gravidade e necessidade de intervenções hospitalares após o parto a fim de garantir a sobrevivência.

De acordo com o artigo 10 da Portaria no 930, de 10 de maio de 2012 20, do Ministério da Saúde (utilizada pelo HC), são alguns motivos para admissão em UTI neonatal: recém-nascidos que necessitem de ventilação mecânica ou estejam em fase aguda de insuficiência respiratória com frações inspiradas de oxigênio maior que 30\%; necessidade de cirurgias de grande porte ou pós-operatório de cirurgias de menor porte; necessidade de cateter venoso central, drogas vasoativas, prostaglandina, uso de antibióticos para tratamento de infecção grave, transfusão de hemoderivados ou distúrbios de coagulação. 
Critérios relacionados ao manejo dos recém-nascidos têm sido propostos para a identificação de casos de near miss neonatal 2,21. Tais discussões atuais refletem a preocupação dos estudiosos no tema quanto à utilidade do conceito para avaliar a qualidade da assistência neonatal. Contudo, os marcadores de manejo se baseiam no modelo de predição de risco para morte, o que não abrange aspectos importantes de avaliação de serviços sob a ótica de processo de trabalho das equipes de saúde, por exemplo. Além disso, a necessidade de consulta a prontuários para análise do universo de nascidos vivos dificulta a sua incorporação na rotina de monitoramento dos casos. A definição SIS construída com dados secundários dos SIS pode contribuir para a vigilância em saúde ao possibilitar um acesso simplificado, com o critério internação em UTI neonatal representando uma proxy para um conjunto de critérios que caracterizam gravidade.

Quanto à comparação do desempenho das definições por meio da validação concorrente, pôde-se observar que a sensibilidade apresentou valores elevados e semelhantes nas três definições quando comparada pelo IC95\%. Por outro lado, a especificidade foi decrescente neste sentido: Silva > PileggiCastro > SIS. O valor preditivo negativo foi próximo a 100\%, similar ao encontrado nos artigos originais dos autores 5,6. $\mathrm{O}$ valor preditivo positivo mais baixo está relacionado à frequência de óbitos neonatais precoces, que é um evento raro do ponto de vista matemático 1.

Recente estudo 22 comparou diferentes definições de near miss neonatal, dentre elas a de Silva et al. 6 e de Pileggi-Castro et al. 5, e restringiu-se aos critérios pragmáticos. Os autores orientam utilizar a definição de Silva por conta da adequação dos pontos de corte com valores usados frequentemente em estudos perinatais, porém reforçam que não foram encontradas diferenças estatisticamente significantes entre as definições utilizadas. Ainda recomendam, quando possível, a obtenção de informações dos recém-nascidos com base no SINASC e do linkage com o SIM, e reconhecem que o uso de critérios de simples coleta nos SIS facilita a operacionalização do conceito de near miss neonatal para fins clínicos e epidemiológicos 22.

Ao se considerar que a sensibilidade está relacionada à proporção de casos que são detectados pelos sistemas de informação, um maior valor significa que a definição pode ser utilizada para triagem, sobretudo se a sensibilidade vier associada a maior classificação de casos como na definição SIS. Dessa forma, além de mais dados a serem coletados, muitos casos de morbidade neonatal precoce que não entrariam por outras definições poderão ser analisados, permitindo maior compreensão de fatores relacionados à saúde dos recém-nascidos com ameaça à vida 2,11,21.

Para ações de vigilância em saúde, o uso de definições que identifiquem o near miss neonatal precoce com maior sensibilidade pode contribuir para evidenciar a existência de possíveis falhas na atenção no pré-natal, durante o parto e no período neonatal precoce, o que não exclui a necessidade de investigação detalhada posterior. O uso do near miss como indicador para monitorar mudanças de situação de morbimortalidade nos estabelecimentos de saúde e identificação de "surtos" ao longo do tempo pode ser um instrumento de gestão decisivo para melhorar a qualidade da assistência neonatal.

Há situações, todavia, em que é preferível utilizar uma definição mais específica. Isso ocorre quando os serviços de saúde não dispõem de capacidade operacional para monitorar um número grande de casos. Nessa situação, a definição Pileggi-Castro pragmática poderia ser utilizada, pois apresentou especificidade e valor preditivo positivo levemente maior e também possui variáveis disponíveis nos SIS.

Como este estudo foi realizado com dados de um único hospital terciário com UTI neonatal que é referência para gestação e parto de alto risco, a população foi mais homogênea e em menor número que a dos artigos originais. Essa pode ser uma justificativa para os maiores valores encontrados e também para os intervalos de confiança mais largos.

A definição proposta por este estudo se mostrou bastante útil para a identificação de casos de near miss neonatal precoce ao apresentar desempenho semelhante às pesquisas mais recentes publicadas na literatura. Os critérios utilizados são de acesso fácil e estão disponíveis nos SIS oficiais do país, fato que permite monitorar recém-nascidos graves pelos próprios estabelecimentos de saúde, uma vez que a coleta de tais dados é uma rotina dos serviços.

Após a superação da mortalidade infantil, surge um desafio maior: a redução do componente neonatal precoce, que está relacionado à qualidade da assistência à gestante e ao recém-nascido desde o período antenatal. A utilidade do near miss neonatal precoce como ferramenta de gestão poderá contribuir para avaliação e identificação de fragilidades na atenção à saúde, bem como para a formação de políticas públicas com foco nesse grupo mais vulnerável. 


\section{Colaboradores}

K. E. X. França contribuiu na concepção e planejamento do estudo, na análise e interpretação dos dados, na elaboração do rascunho e aprovação da versão final do manuscrito. M. B. R. Vilela contribuiu na concepção e planejamento do estudo, na análise e interpretação dos dados e na aprovação da versão final do manuscrito. P. G. Frias e G. S. Gaspar contribuíram na análise e interpretação dos dados, na elaboração do rascunho e aprovação da versão final do manuscrito. S. W. Sarinho contribuiu na concepção e planejamento do estudo, na interpretação dos dados e aprovação da versão final do manuscrito.

\section{Agradecimentos}

À Dra. Lindacir Sampaio, coordenadora da unidade neonatal do Hospital das Clínicas da Universidade Federal de Pernambuco, pela ajuda e pelos esclarecimentos durante a pesquisa. Ao Prof. Paulo Goes pelas suas contribuições metodológicas.

\section{Referências}

1. Pileggi C, Souza JP, Cecatti JG, Faúndes A. Neonatal near miss approach in the 2005 WHO Global Survey Brazil. J Pediatr (Rio J.) 2010; 86:21-6.

2. Santos J, Cecatti J, Serruya S, Almeida P, Duran P, Mucio B, et al. Neonatal near miss: the need for a standard definition and appropriate criteria and the rationale for a prospective surveillance system. Clinics 2015; 70:820-6.

3. Avenant T. Neonatal near miss: a measure of the quality of obstetric care. Best Pract Res Clin Obstet Gynaecol 2009; 23:369-74.

4. Say L, Souza JP, Pattinson RC. Maternal near miss - towards a standard tool for monitoring quality of maternal health care. Best Pract Res Clin Obstet Gynaecol 2009; 23:287-96.

5. Pileggi-Castro C, Camelo JS, Perdoná GC, Mussi-Pinhata MM, Cecatti JG, Mori R, et al. Development of criteria for identifying neonatal near-miss cases: analysis of two WHO multicountry cross-sectional studies. BJOG 2014;121 Suppl:110-8.

6. Silva AAM, Leite AJM, Lamy ZC, Moreira MEL, Gurgel RQ, Cunha AJLA, et al. Morbidade neonatal near miss na pesquisa Nascer no Brasil. Cad Saúde Pública 2014; 30 Suppl:S182-91.

7. Ronsmans C, Cresswell JA, Goufodji S, Agbla S, Ganaba R, Assarag B, et al. Characteristics of neonatal near miss in hospitals in Benin, Burkina Faso and Morocco in 2012-2013. Trop Med Int Health 2016; 21:535-45.

8. Frias PG, Szwarcwald CL, Lira PIC. Avaliação dos sistemas de informações sobre nascidos vivos e óbitos no Brasil na década de 2000. Cad Saúde Pública 2014; 30:2068-80.

9. Pereira CCB, Vidal SA, Carvalho PI, Frias PG. Avaliação da implantação do Sistema de Informações sobre Nascidos Vivos (SINASC) em Pernambuco. Rev Bras Saúde Matern Infant 2013; 13:39-49. 
10. Lansky S, Friche AADL, Silva AAM, Campos D, Bittencourt SDDA, Carvalho ML, et al. Pesquisa Nascer no Brasil: perfil da mortalidade neonatal e avaliação da assistência à gestante e ao recém-nascido. Cad Saúde Pública 2014; 30 Suppl :S192-207.

11. Kale PL, Mello-Jorge MHP, Silva KS, Fonseca SC. Casos de near miss e óbitos neonatais: fatores associados aos recém-nascidos com ameaça à vida em seis maternidades do Sudeste do Brasil Cad Saúde Pública 2017; 33:e00179115.

12. Ministério da Educação, Hospitais Universitários Federais. HC em números. Hospital das Clínicas, UFPE. http://www.ebserh.gov.br/ web/hc- ufpe/licitacoes-e-contratacoes (acessado em 15/Nov/2016).

13. Landis JR, Koch GG. The measurement of observer agreement for categorical data. Biometrics 1977; 33:159-74.

14. Fleiss J. Statistical methods for rates and proportions. 2nd Ed. New York: John Wiley \& Sons; 1981.

15. Silva RS, Oliveira CM, Ferreira DKS, Bonfim CV. Avaliação da completitude das variáveis do Sistema de Informações sobre Nascidos Vivos - SINASC - nos estados da região Nordeste do Brasil, 2000 e 2009. Epidemiol Serviços Saúde 2013; 22:347-52.

16. Pereira APE, Dias MAB, Bastos MH, Gama SGN, Leal MC. Determining gestational age for public health care users in Brazil: comparison of methods and algorithm creation. BMC Res Notes 2013; 6:60.
17. Berhan Y, Berhan A. Review a meta-analysis of selected maternal and fetal factors for perinatal mortality. Ethiop J Health Sci 2014; 24:55-68.

18. Gonzaga ICA, Santos SLD, Silva ARV, Campelo V. Atenção pré-natal e fatores de risco associados à prematuridade e baixo peso ao nascer em capital do Nordeste brasileiro. Ciênc Saúde Coletiva 2016; 21:1965-74.

19. Silva CF, Leite AJM, Almeida NMGS, Ponce de Leon ACM, Olofin I; Rede Norte-Nordeste de Saúde Perinatal. Fatores associados ao óbito neonatal de recém-nascidos de alto risco: estudo multicêntrico em Unidades Neonatais de Alto Risco no Nordeste brasileiro. Cad Saúde Pública 2014; 30:355-68.

20. Ministério da Saúde. Portaria no 930, de 10 de maio de 2012. Define as diretrizes e objetivos para a organização da atenção integral e humanizada ao recém-nascido grave ou potencialmente grave e os critérios de classificação. Diário Oficial da União 2012; 11 mai.

21. Santos JP, Pileggi-Castro C, Camelo JS, Silva AA, Duran P, Serruya SJ, et al. Neonatal near miss: a systematic review. BMC Pregnancy Childbirth 2015; 15:320.

22. Kale PL, Melo-Jorge MHPM, Laurenti R, Fonseca SC, Silva KS. Pragmatic criteria of the definition of neonatal near miss: a comparative study. Rev Saúde Pública 2017; 51:111. 


\section{Abstract}

The aim of this study was to validate a definition to identify cases of early neonatal near miss using data from health information systems (SIS in Portuguese). This was a concurrent validation study focusing on three definitions for identification of cases of early neonatal near miss among live births in a university hospital in 2012. Three different definitions were applied to this live birth cohort using the criteria birth weight, gestational age, 5-minute Apgar score, admission to the neonatal intensive care unit, mechanical ventilation, and congenital malformations, in different combinations, considering the proposals in two Brazilian articles (Silva et al.; Pillegi-Castro et al.) and a third (SIS definition) with available data from health information systems. Cases were defined as infants that had survived the risk conditions as of the 7th day of life. For concurrent validation, the study adopted early neonatal deaths as the reference. Of the 2,097 live births studied, 33 died in the early neonatal period, and the number of cases of early neonatal near miss varied according to the definition used: 153 (Silva definition), 194 (Pileggi-Castro definition), and 304 (SIS definition). Sensitivity and specificity were $97 \%$ and $92.6 \%$, respectively, according to the Silva definition, 90.9\% and $90.6 \%$ according to the Pileggi-Castro definition, and $93.9 \%$ and $85.3 \%$ according to the SIS definition. The results show that the SIS definition has sensitivity and specificity close to the other definitions and suggest that it is possible to monitor early neonatal near miss using only data that are available in official health information systems.

Healthcare Near Miss; Newborn Infant; Early Neonatal Mortality; Vital Statistics; Information Systems

\section{Resumen}

El objetivo fue validar una definición de identificación de casos de near miss neonatales precoces, utilizando datos de los sistemas de información en salud (SIS). Se trata de un estudio de validación concurrente entre tres definiciones para la identificación de casos de near miss neonatales precoces, realizado en un hospital universitario, con nacidos vivos que se produjeron en 2012. Se aplicaron tres definiciones a esta cohorte de nacidos vivos con la utilización de los criterios: peso al nacer, edad gestacional, índice de Apgar en el 50 minuto de vida, internamiento en la Unidad de Terapia Intensiva Neonatal, ventilación mecánica, además de malformaciones congénitas con diferentes combinaciones, considerando las propuestas de dos artículos brasileños publicados (definición Silva et al.; definición Pillegi-Castro et al.) y una tercera (definición SIS) con datos disponibles en el Sistema de Información en Salud. Se consideraron casos los supervivientes en condiciones de riesgo hasta el 7o día de vida. Para la validación concurrente, se adoptaron como referencia los óbitos neonatales precoces. De los 2.097 nacidos vivos estudiados, 33 fueron óbito durante el período neonatal precoz, y el número de casos de near miss neonatal precoz varió según la definición adoptada: 153 (definición Silva), 194 (definición PileggiCastro) y 304 (definición SIS). La sensibilidad y especificidad fue, respectivamente, 97\% y 92,6\% en la definición Silva, 90,9\% y 90,6\% en la definición Pileggi-Castro y 93,9\% y 85,3\% en la definición SIS. Los resultados muestran que la definición SIS presenta sensibilidad y especificidad próxima a las otras definiciones y sugiere que es posible monitorear el near miss neonatal precoz sólo con el uso de datos disponibles en los Sistemas oficiales de Información en Salud.

Near Miss Salud; Recién Nacido; Mortalidad Neonatal Precoz; Estadística Vitales; Sistemas de Información
Recebido em 25/Set/2017

Versão final reapresentada em 05/Abr/2018

Aprovado em 13/Abr/2018 